Supporting Information for:

\title{
Visible-to-Ultraviolet Upconversion Efficiency above $10 \%$ Sensitized by Quantum-Confined Perovskite Nanocrystals
}

Shan He, Xiao Luo, Xue Liu, Yulu Li and Kaifeng Wu*

${ }^{\dagger}$ State Key Laboratory of Molecular Reaction Dynamics and Dynamics Research

Center for Energy and Environmental Materials, Dalian Institute of Chemical

Physics, Chinese Academy of Sciences, Dalian, Liaoning 116023, China

* Corresponding Author: kwu@dicp.ac.cn

\section{Content list:}

Figures S1-S6

Sample preparations

TTA-UC measurements

Time-resolved spectroscopy measurements

Kinetics fitting

Table S1, S2 


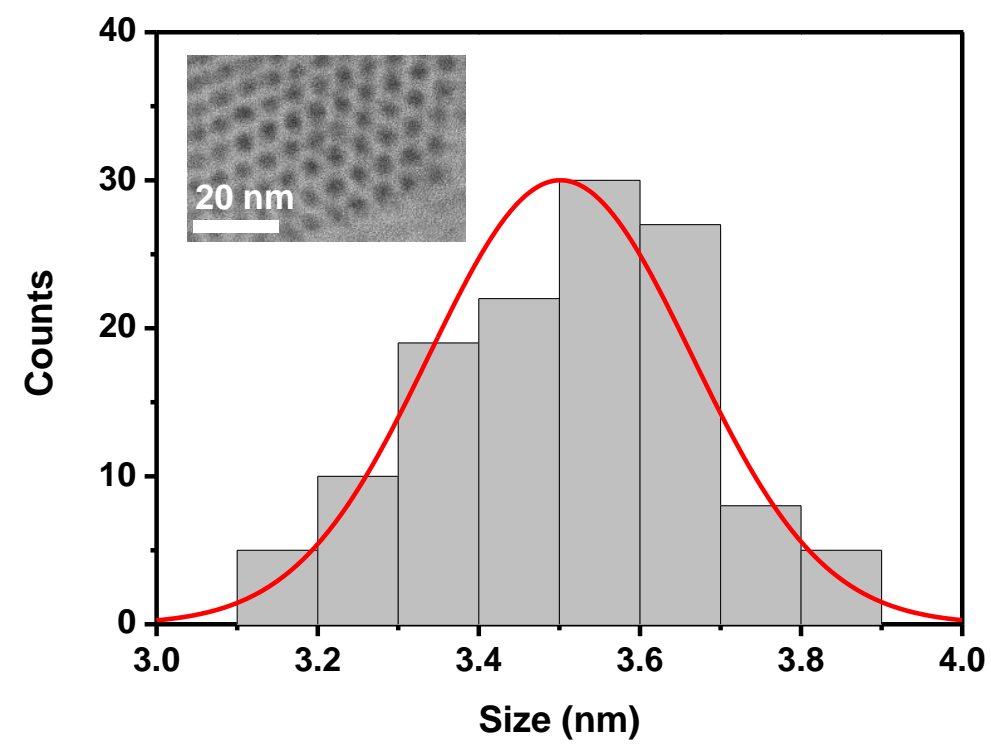

Figure S1. TEM image of the $\mathrm{CsPbBr}_{3} \mathrm{NCs}$ used in this study.
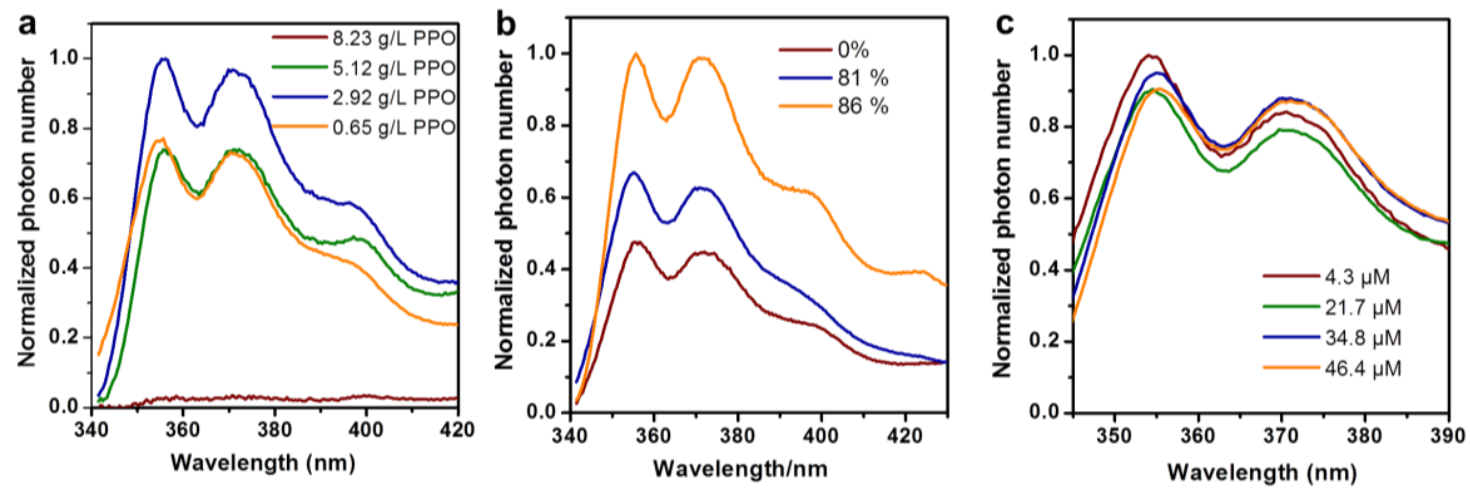

Figure S2. TTA-UC emission spectra using (a) different PPO concentrations, (b) different hexane proportions (the solvent is the mixture of hexane and n-octane), and (c) different $\mathrm{NC}$ concentrations. The use of hexane as the solvent increases the UC efficiency for two reasons. First, the NCA ligands have a lower solubility in hexane $(<0.1 \mathrm{mM})$ than in octane $(\sim 3 \mathrm{mM})$; as such, NCA binds more strongly to NC surfaces in hexane, which enables more efficient TET from NCs to NCA. Secondly, hexane 
has a lower viscosity than octane $(0.30 v s \quad 0.55 \mathrm{mPa} \cdot \mathrm{s})$, allowing more efficient TET from NCA to PPO and TTA between PPO via diffusion. ${ }^{1}$

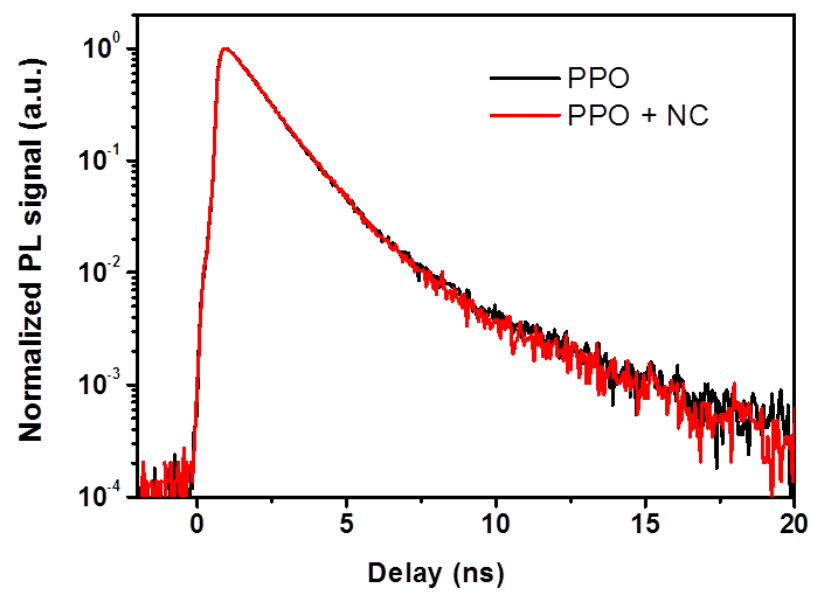

Figure S3. The PL kinetics of PPO excited states (monitored at $372 \mathrm{~nm}$; excited at $316 \mathrm{~nm}$ ) measured in the absence (black) and presence (red) of $\mathrm{CsPbBr}_{3} \mathrm{NCs}$. The kinetics are similar, suggesting that energy transfer from PPO excited states to NCs is much slower than their intrinsic decay. 
fiber

spectrometer

lens system

laser
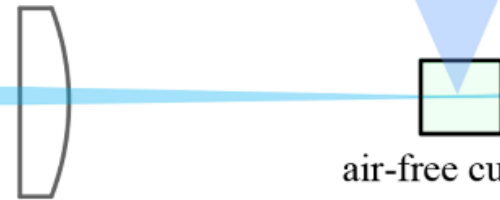

air-free cuvette

lens

Figure S4. Apparatus for detecting upconversion emission.

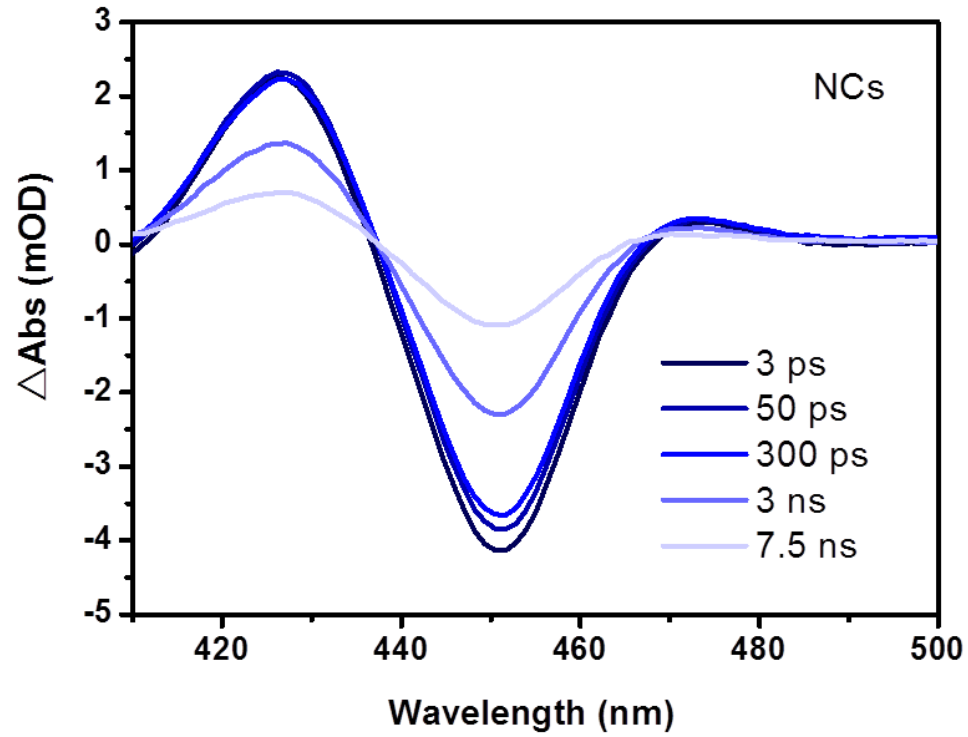

Figure S5. TA spectra of free NCs at selective time delays following the excitation by a $400 \mathrm{~nm}$ pulse. 


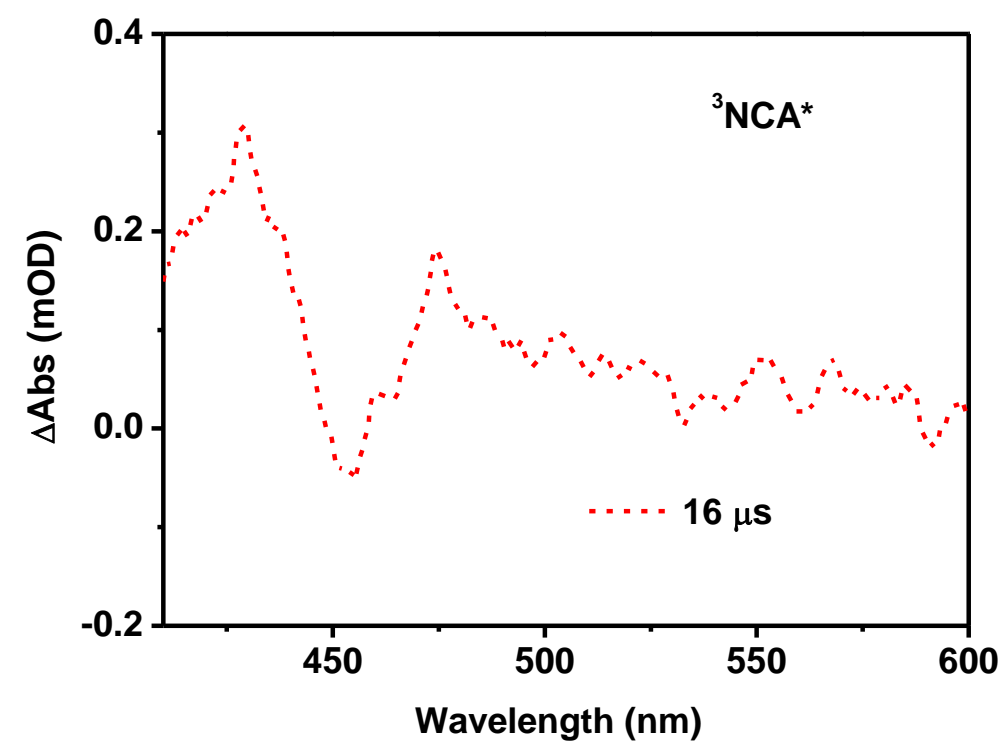

Figure S6. TA spectrum of the NC-NCA sample at a delay of $16 \mu$ s showing the $\mathrm{T}_{1} \rightarrow$ $\mathrm{T}_{\mathrm{n}}$ absorption of NCA triplets $\left({ }^{3} \mathrm{NCA}^{*}\right)$. The dip at $\sim 450 \mathrm{~nm}$ is likely due to the exciton bleach of NCs generated through delayed reverse TET from NCA to NCs.

\section{Sample preparations}

Synthesis of $\mathrm{CsPbBr}_{3} \mathrm{NCs}$. $\mathrm{CsPbBr}_{3}$ perovskite NCs were synthesized following previously reported procedures. ${ }^{2}$ Briefly, $0.25 \mathrm{~g} \mathrm{Cs} 2 \mathrm{CO}_{3}, 0.8 \mathrm{~g}$ oleic acid (OA), and $7 \mathrm{~g}$ 1-octadecene (ODE) were loaded into a $25 \mathrm{~mL}$ 3-neck flask and vacuum-dried for $1 \mathrm{~h}$ at $120^{\circ} \mathrm{C}$ using a Schlenk line. The mixture was heated under Ar atmosphere to 150 ${ }^{\circ} \mathrm{C}$ until all the $\mathrm{Cs}_{2} \mathrm{CO}_{3}$ powders were dissolved. The Cs-oleate precursor solution was kept at $100{ }^{\circ} \mathrm{C}$ to prevent precipitation of Cs-oleate out of ODE. In another $50 \mathrm{~mL}$ 3-neck flask, the precursor solution of $\mathrm{Pb}$ and $\mathrm{Br}$ was prepared by dissolving $228 \mathrm{mg}$ $\mathrm{PbBr}_{2}$ and $\mathrm{ZnBr}_{2}(603 \mathrm{mg})$ in a mixture of $\mathrm{ODE}(15 \mathrm{~mL}), \mathrm{OA}(8 \mathrm{~mL})$, and oleylamine 
(OAm, $8 \mathrm{~mL}$ ). After the precursor solution of $\mathrm{Pb}$ and $\mathrm{Br}$ were vacuum-dried for $1 \mathrm{~h} \mathrm{at}$ $120^{\circ} \mathrm{C}$ and reached $87^{\circ} \mathrm{C}$ under Ar atmosphere, $1.2 \mathrm{~mL}$ of $\mathrm{Cs}$ precursor solution was injected to initiate the reaction. The reaction was quenched after a short period of time (90 s) by cooling the flask in an ice bath. After the crude solution was cooled down to room temperature, the product was centrifuged at $4500 \mathrm{rpm}$ for $5 \mathrm{~min}$ to remove the unreacted salts asthe precipitate, and the perovskite NCs dispersed in the supernatant were collected. NCs were precipitated by adding acetone dropwise until the mixture just turned turbid to avoid decomposition of the NCs. The dried NCs were collected and dissolved in hexane.

Preparation of $\mathrm{CsPbBr}_{3}$ NCs-NCA complexes. The $\mathrm{CsPbBr} 3$ NCs-NCA complexes were prepared by simply adding 1-naphthalene carboxylic acid (NCA) powders into the $\mathrm{CsPbBr} 3 \mathrm{NCs}$ solution followed by sonication and filtration. Because the solubility of NCA molecules in hexane or n-octane is negligible, all the molecules remaining in the solution after filtration were believed to be bound to NC surfaces.

\section{TTA-UC measurements}

The samples were prepared in glovebox and sealed in custom-made air-free quartz cuvettes. Photoluminescence quantum yields were determined relative to coumarin 307 in ethanol $\left(\Phi_{\mathrm{C} 307}=75 \%\right)$.

The fluorescence detecting apparatus is shown in Figure S3. The sample was excited by a $443 \mathrm{~nm} \mathrm{CW}$ laser. The laser beam was focused by a quartz lens. The focal point was in the air-free cuvettes. Along the axis perpendicular to the excitation laser, a lens 
system was used to collect the fluorescence and couple it into a fiber. The other side of the fiber was connected to a spectrometer (Maya2000 pro; Ocean Optics). The wavelength-dependent response of the spectrometer is calibrated by using laser light from a TOPAS Optical Parametric Amplifier (OPA) pumped by a femtosecond amplifier. Specifically, output lights with continuously tunable wavelength and known power densities were used to calculate the photon numbers and to calibrate the response of the spectrometer.

The upconversion quantum yield ( $\left.\Phi^{\prime}{ }_{U C}\right)$ was calculated according to eq. (S1),

$$
\Phi^{\prime}{ }_{\mathrm{UC}}=2 \Phi_{\mathrm{C} 307} \frac{\left(1-10^{-\mathrm{A}_{\mathrm{C} 307}}\right)}{\left(1-10^{-\mathrm{A}_{\mathrm{S}}}\right)} \frac{\mathrm{F}_{\mathrm{S}}}{\mathrm{F}_{\mathrm{C} 307}} \frac{\mathrm{n}_{\mathrm{S}}^{2}}{\mathrm{n}_{\mathrm{C} 307}^{2}}
$$

Where $A_{i}$ is the absorption (optical density) at the excitation wavelength, $F_{i}$ is the integrated photon number and $\mathrm{n}_{\mathrm{i}}$ is the refractive index of the solvent. Subscripts C307 and S are coumarin 307 and sample, respectively. Herein we have chosen to report the normalized upconversion quantum yield (theoretical maximum being $100 \%)$. The data for upconversion quantum yield of $10.2 \%$ were listed in Table S1.

Table S1. Data for upconversion quantum yield. The same sample was measured twice with excitation laser power density of 58.8 (top) and 51.5 (bottom) W/cm², respectively.

\begin{tabular}{cccccc}
\hline & \multirow{2}{*}{ Abs } & \multicolumn{2}{c}{ photon number } & \multicolumn{2}{c}{ quantum yield } \\
\cline { 3 - 6 } & & band edge & upconversion & band edge & upconversion \\
\hline Coumarin 307 & 0.18 & 51222.4 & - & $75.0 \%$ & - \\
$\mathrm{NC}$ & 0.35 & 68764.5 & - & $62.2 \%$ & - \\
$\mathrm{NC}+\mathrm{NCA}+\mathrm{PPO}$ & 0.36 & 11168.2 & 5730.2 & $9.9 \%$ & $10.2 \%$ \\
\hline
\end{tabular}




\begin{tabular}{cccccc}
\cline { 3 - 4 } Coumarin 307 & 0.18 & 52190.9 & - & $75.0 \%$ & - \\
$\mathrm{NC}$ & 0.35 & 69041.9 & - & $61.3 \%$ & - \\
$\mathrm{NC}+\mathrm{NCA}+\mathrm{PPO}$ & 0.36 & 11158.5 & 5800.0 & $9.7 \%$ & $10.1 \%$ \\
\hline
\end{tabular}

\section{Time-resolved spectroscopy measurements}

Transient absorption (TA). The femtosecond pump-probe TA measurements were performed using a regenerative amplified Ti:sapphire laser system (Coherent; $800 \mathrm{~nm}$, $70 \mathrm{fs}, 6 \mathrm{~mJ} /$ pulse, and $1 \mathrm{kHz}$ repetition rate) as the laser source and a femto-100 spectrometer (Time-Tech LLC). Briefly, the $800 \mathrm{~nm}$ output pulse from the regenerative amplifier was split in two parts with a 50\% beam splitter. The transmitted part was used to pump a TOPAS Optical Parametric Amplifier (OPA) which generated a wavelength-tunable laser pulse from $250 \mathrm{~nm}$ to $2.5 \mu \mathrm{m}$ as pump beam. The reflected $800 \mathrm{~nm}$ beam was split again into two parts. One part with less than $10 \%$ was attenuated with a neutral density filter and focused into a crystal to generate a white light continuum (WLC) used for probe beam. The probe beam was focused with an Al parabolic reflector onto the sample. After the sample, the probe beam was collimated and then focused into a fiber-coupled spectrometer with CMOS sensors and detected at a frequency of $1 \mathrm{KHz}$. The intensity of the pump pulse used in the experiment was controlled by a variable neutral-density filter wheel. The delay between the pump and probe pulses was controlled by a motorized delay stage. The pump pulses were chopped by a synchronized chopper at $500 \mathrm{~Hz}$ and the absorbance change was calculated with two adjacent probe pulses (pump-blocked and pump-unblocked). The samples were placed in $1 \mathrm{~mm}$ airtight cuvettes in a $\mathrm{N}_{2}$-filled glove box and measured 
under ambient conditions.

Nanosecond TA was performed with the EOS spectrometer (Ultrafast Systems LLC). The pump beam is generated in the same way as the femtosecond TA experiment described above. A different white light continuum (380-1700 nm, $0.5 \mathrm{~ns}$ pulse width, $20 \mathrm{kHz}$ repetition rate) was used, which was generated by focusing a Nd:YAG laser into a photonic crystal fiber. The delay time between the pump and probe beam was controlled by a digital delay generator (CNT-90, Pendulum Instruments).

Time-resolved PL. Time-resolved PL decay was measured using time-correlated single-photon counting (TCSPC) set-up. The excitation source was a Pharos laser (1030 nm,100 kHz, 230 fs pulse-duration; Light conversion), which was used to pump a TOPAS OPA to generate wavelength-tunable excitation pulses. All samples were placed in $1 \mathrm{~mm}$ airtight cuvettes in a $\mathrm{N}_{2}$-filled glove box and were vigorously stirred in all the measurements.

\section{Kinetics fitting}

The decay curve for free NCs can be fitted by a single-exponential decay function, whereas that of the NC-NCA sample needs to be fitted by a 2-exponential function. The average lifetime $\tau_{\text {ave }}$ was calculated according to eq. (S2),

$$
\tau_{\mathrm{ave}}=\frac{\mathrm{A}_{1} \tau_{1}+\mathrm{A}_{2} \tau_{2}}{\mathrm{~A}_{1}+\mathrm{A}_{2}}
$$

where $\mathrm{A}_{\mathrm{i}}$ and $\tau_{\mathrm{i}}$ are the amplitude and lifetime, respectively, of the $\mathrm{i}$-th exponential component. 
The lifetime of NCs is $\tau_{\mathrm{NC}}$ and the decay rate can be described as $\frac{1}{\tau_{\mathrm{NC}}}$. Similarly, the decay rate of NCs-NCA can be described as $\frac{1}{\tau_{\text {ave }}}$. Therefore, the TET rate is $\frac{1}{\tau_{\mathrm{ave}}}-\frac{1}{\tau_{\mathrm{NC}}}$ and TET yield is $1-\frac{\tau_{\mathrm{ave}}}{\tau_{\mathrm{NC}}}$

Table S2. Kinetics fitting parameters

\begin{tabular}{|c|c|c|c|c|c|c|}
\hline & \multicolumn{2}{|c|}{$\tau(\mathrm{ns})$} & \multirow{2}{*}{$\mathrm{A}_{1}(\%)$} & \multirow{2}{*}{$\mathrm{A}_{2}(\%)$} & \multirow{2}{*}{$\tau_{\text {ave }}(n s)$} & \multirow{2}{*}{$\Phi_{\mathrm{TET}}(\%)$} \\
\hline & $\tau_{1}(\mathrm{~ns})$ & $\tau_{2}(\mathrm{~ns})$ & & & & \\
\hline $\mathrm{NCs}$ & \multicolumn{2}{|c|}{$5.93 \pm 0.07$} & & & - & \\
\hline NCs-NCA & $1.18 \pm 0.04$ & $0.13 \pm 0.02$ & 84.0 & 16.0 & $1.02 \pm 0.03$ & 82.8 \\
\hline
\end{tabular}

\section{References for SI.}

(1) Huang, Z.; Li, X.; Yip, B. D.; Rubalcava, J. M.; Bardeen, C. J.; Tang, M. L. Nanocrystal Size and Quantum Yield in the Upconversion of Green to Violet Light with CdSe and Anthracene Derivatives. Chem. Mat. 2015, 27, 7503-7507.

(2) Dong, Y.; Qiao, T.; Kim, D.; Parobek, D.; Rossi, D.; Son, D. H. Precise Control of Quantum Confinement in Cesium Lead Halide Perovskite Quantum Dots via Thermodynamic Equilibrium. Nano Lett. 2018, 18, 3716-3722. 